\title{
Quelques limitations théoriques des procédés de visualisation
}

\section{The theoretical limitation of visual observation methods}

\author{
PAR J. FAURE,

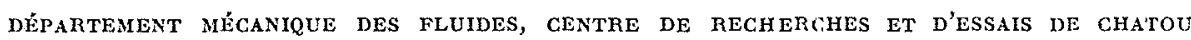

\begin{abstract}
Après un examen des principales méthodes de visnalisation, il est possible d'indiquer les limites que la mécanique des fluides impose (masse ajoutée - différence de densité - turbulence - diffusion).
\end{abstract}

\begin{abstract}
An examination of the principal methods of visual observation provides an indication of the limitations due to factors of fluid mechanics (e.g. added mass, density difference, turbulence, diffusion).
\end{abstract}

\section{1. - PRINCIPALES MÉTHODES DE VISUALISATION}

Aux vitesses modérées des fluides les principales méthodes de visualisation sont les suivantes :

\subsection{Méthodes optiques.}

Elles sont de deux natures, la méthode des ombres et l'interférométrie.

\section{a) Méthode des ombres.}

Elle utilise la réfraction de la lumière à la surface libre d'un liquide, et l'intensité de la lumière qui atteint une plaque sensible dépend de la courbure de la surface libre du liquide, donc de la dérivée seconde de l'équation de la surface libre. En coordonnées bidimensionnelles, si la surface libre a pour équation $y=f(x)$,

- pour $\frac{d^{2} y}{d x^{2}}=0$ ou $\frac{d y}{d x}=$ Cte, l'intensité de la lumière ne varie pas;

- pour $\frac{d^{2} y}{d x^{2}}>0$ (surface convexe vers le haut), l'image présente une intensité lumineuse plus grande (les rayons convergent);

- pour $\frac{d^{2} y}{d x^{2}}<0$ (surface concave vers le haut), il y a réduction de l'intensité lumineuse (les rayons divergent).
Cette méthode s'utilise pour les écoulements à surface libre qui peuvent par analogie représenter des écoulements compressibles.

b) IN'TERFÉROMÉTRIE.

Cette méthode est basée sur la variation d'indice de réfraction du fluide avec la variation de densité liée aux variations de pression, de températures, aux échanges thermiques, aux variations de concentrations (dans les réactions chimiques par exemple). Dans le fluide, la déviation des rayons lumineux et leur intensité sont proportionnelles au gradient de densité du fluide. Par suite la région où les rayons lumineux sont déviés apparaîtra en sombre sur les clichés.

\subsection{Eléments traçants.}

Avec les fluides courants (eau et air) les éléments traçants utilisés sont variés; on utilise: - des poudres (métaux, en particulier aluminium, ou poudre de balsa ou polystyrène);

- des bulles d'air ou des gouttes d'huile;

- des fumées ou des liquides colorés.

La technique d'étude consiste à éclairer fortement une tranche de l'écoulement et à observer photographiquement le déplacement des par- 
ticules et les expérimentateurs savent que la qualité des résultats croît lorsque la différence de densité entre fluide et éléments tracants diminue et lorsque la taille des particules diminue à un point tel qu'elles deviennent individuellement invisibles.

\subsection{Traitement de surface.}

Deux techniques principales sont utilisées :

- filaments séchés;

- revêtement de surfaces.

Les petits filaments de soie ou de coton placés en un point de l'écoulement ont des mouvements liés à la turbulence de l'écoulement; s'ils sont enduits d'un liquide plastique qui durcit au séchage, leur position finale schématise l'allure de l'écoulement.

De même, pour les écoulements près des surfaces (problèmes de couches limites en particulier), on recouvre les surfaces de mélanges divers qui, en séchant, matérialisent l'écoulement.

\subsection{Domaine d'emploi.}

Toutes les méthodes précédentes ont été perfectionnées et fournissent des indications intéressantes dans des problèmes particuliers pour lesquels elles sont adaptées. Lorsque les accélérations ou les courbures ne sont pas fortes et lorsque les dimensions des éléments tracants, par exemple, sont suffisamment petites, elles donnent d'excellents résultats et constituent même des moyens de mesure.

Par contre, et c'est cela qui est intéressant, lorsque les écoulements sont fluctuants ou très éloignés des conditions uniformes, la visualisation par ces procédés donnerait une image fausse de l'écoulement. Dans la suite, nous nous attacherons particulièrement au problème des éléments traçants et nous indiquerons les procédés de calcul qui permettent de déterminer leur nature et leurs dimensions de facon que la visualisation soit représentative de l'écoulement et puisse conduire à des valeurs numériques.

\section{2. - LIMITATIONS THEORIQUES}

\subsection{Généralités.}

A part les procédés optiques, les méthodes expérimentales d'étude des fluides conduisent toutes à une perturbation de l'écoulement, soit par l'introduction d'appareillage de mesure, soit par l'introduction d'éléments traçants et on peut donner pour les fluides un principe analogue au principe d'incertitude d'Heisenberg : ou bien on connaît exactement la position d'un point de mesure (cas de l'introduction d'un appareillage de mesure), mais on perturbe l'écoulement et la mesure, même précise, donne un indication différente de celle que l'on veut mesurer; ou bien on perturbe moins l'écoulement par introduction d'éléments traçants de faibles dimensions, mais la mesure (sous réserve des restrictions ultérieures) ne peut pas être rapportée à un point précis.

Les principales limitations des procédés de visualisation sont les suivantes :

- la visualisation change les caractéristiques du fluide (structure - densité - viscosité);

- la plupart des méthodes sont basées sur des mesures optiques qui, sauf complications expérimentales, sont bidimensionnelles;

- les éléments traçants fournissent sur les clichés des trajectoires (un calcul est alors nécessaire pour retrouver les lignes de courant);
- les fumées ou liquides colorés donnent des lignes d'émission.

Dans les problèmes envisagés, trajectoires, lignes de courant et lignes d'émission sont différentes et nous allons indiquer ci-après l'influence des caractéristiques non uniformes ou non permanentes de l'écoulement sur les éléments traçants.

La qualité de la visualisation par particules traçantes a été définie par M. Bourot [1] par les deux paramètres $\alpha$, d'écart angulaire et $\lambda$, de glissement, tels que :

$$
\begin{aligned}
& \alpha=\left(\overrightarrow{\mathrm{V}}_{p}, \overrightarrow{\mathrm{V}}_{\mathrm{F}}\right) \\
& \lambda=\frac{\nabla_{p}-V_{\mathrm{F}}}{\bar{V}_{p}}
\end{aligned}
$$

que M. Bourot relie aux caractéristiques de la particule :

$$
\begin{aligned}
& \alpha=\frac{1}{\mathrm{~K}}\left(\frac{\mathrm{V}}{\mathrm{R}}\right)_{p} \\
& \lambda=-\frac{1}{\mathrm{~K}}\left(\frac{d \mathrm{~V}}{d s}\right)_{p}
\end{aligned}
$$

K, coefficient caractérisant particules et fluides et toutes les autres variables étant rapportées à la particule. 


\subsection{Effet de masse ajoutée.}

On sait que lorsqu'un corps de masse $m$ se déplace d'un mouvement rectiligne uniforme à une vitesse $v$, son énergie cinétique est:

$$
\mathrm{E}_{c}=\frac{1}{2} m v^{2}
$$

Si le corps se meut à la vitesse $v$ dans un fluide parfait incompressible infini au repos à l'infini et irrotationnel, l'énergie cinétique totale du fluide est :

$$
\mathbf{E}_{f}=\frac{1}{2} c v^{2}
$$

où $c$ est une constante qui ne dépend que de la densité du fluide, la taille et la forme du corps
Lorsqu'on écrit l'équation du mouvement d'une particule dans un fluide, équation de la forme :

$$
\mathrm{M} \frac{d v}{d t}=\mathrm{F}-\mathrm{R}(\mathrm{R} \text { terme de frottement })
$$

le terme $\mathrm{M}$ doit être égal à la masse totale (masse réelle + masse d'inertie); pour une particule sphérique, $c$ est de l'ordre de la moitié de la masse fluide déplacée, on peut dans ce cas écrire :

$$
\mathrm{M}=\rho_{p} \frac{4}{3} \pi \mathrm{R}^{3}+\frac{1}{2} \frac{4}{3} \rho_{f} \pi \mathrm{R}^{3}=\mathrm{M}_{p}+\mathrm{M}_{f}=\mathrm{M}_{p} \mathrm{~S}
$$

\begin{tabular}{|c|c|c|c|c|}
\hline \multicolumn{2}{|c|}{ Nature du fluide } & \multicolumn{2}{|c|}{ NatUre de la particule } & \multirow[t]{2}{*}{ Valeur de $M$} \\
\hline & $\rho_{f}$ & & $\rho_{p}$ & \\
\hline eaut & 1000 & $\begin{array}{l}\text { eau-huile } \\
\text { solide } \\
\text { air }\end{array}$ & $\begin{array}{rrrr}900 \text { à } & 1 & 000 \\
1000 & \text { à } & 5 & 000 \\
& 1 & \end{array}$ & $\begin{array}{c}1,5 \mathrm{M}_{f} \\
1,5 \stackrel{\dot{a}}{5,5} \mathrm{M}_{f} \\
0,5 \mathrm{M}_{f}\end{array}$ \\
\hline air & 1 & $\begin{array}{c}\text { eau } \\
\text { solide }\end{array}$ & $\begin{array}{c}1000 \\
1000 \text { à } 5000\end{array}$ & $\begin{array}{c}1000 \mathrm{M}_{f} \\
1000 \text { à } 5000 \mathrm{M}_{f}\end{array}$ \\
\hline
\end{tabular}

La particule solide aura, toutes choses égales par ailleurs, un mouvement voisin de la particule fluide si $\mathrm{M} \# \mathrm{M}_{f}$; d'où les cas suivants. et la direction du mouvement. L'énergie cinétique totale du système est :

$$
\mathrm{E}=\mathrm{E}_{c}+\mathrm{E}_{f}=\frac{1}{2}(m+c) v^{2}
$$

En période d'accélération, la variation d'énergie cinétique totale du système est:

$$
\frac{d \mathrm{E}}{d t}=(m+c) v \frac{d v}{d t}
$$

qui est égale par définition à $F v$ (F résultante des forces extérieures qui agissent sur le système) d'où :

$$
\mathrm{F}=(m+c) \frac{d v}{d t}
$$

Ainsi, la présence du fluide a pour effet d'accroître l'inertie totale du corps. Le calcul complet est donné dans la référence [2] qui précise les hypothèses du calcul. Il existe sur le sujet une très importante littérature technique anglosaxonne.
Donc, aucune particule traçante ne donne satisfaction dans l'air. Dans l'eau, on peut réaliser des particules d'air chargées qui donnent $\mathrm{M}=1$ (cas de la flottation).

$\mathrm{M}=\mathrm{M}_{p} \mathrm{~S}\left(\mathrm{M}_{p}\right.$ masse de la particule), avec

$$
\begin{aligned}
& \mathrm{S}=\left(1+\frac{1}{2} \frac{\rho_{f}}{\rho_{p}}\right) \text { sphère, } \\
& \mathrm{S}=\left[1+\frac{8}{3 \pi} \frac{a}{h} \frac{\rho_{f}}{\rho_{p}}\right]
\end{aligned}
$$

disque de rayon a d'épaisseur $h$,

d'autres effets sont à inclure et, pour des particules d'aluminium, on a :

$$
\begin{aligned}
\mathrm{S} & =1,0024 \text { sphère dans l'air, } \\
& =1,185 \quad \text { sphère dans l'eau, } \\
& =1,002 \quad \text { lamelles dans l'air }(h / 2 a=0,1), \\
& =2,57 \quad \text { lamelle dans l'eau }(h / 2 a=0,1) .
\end{aligned}
$$

Dans le cas de plusieurs particules en présence, les résultats précédents sont modifiés. 


\subsection{Effet d'une discontinuité de pression.}

Dans une discontinuité de pression (front de flamme, onde de choc), le fluide est violemment accéléré et une particule étrangère est accélérée de facon différente. Dans une discontinuité de pression $(\Delta \mathrm{P})$ une particule prismatique de base $A$ de longueur $h$ subit l'accélération :

$$
\mathrm{M} \frac{d^{2} x}{d t^{2}}=\mathrm{A} \Delta \mathrm{P}
$$

en négligeant forces visqueuses, accélération du fluide déplacé et force extérieure. La relation donnant la variation d'énergie cinétique de la particule est :

$$
\frac{1}{2} \mathrm{M} u_{2}^{2}=\frac{1}{2} \mathrm{M} u_{1}^{2}+\mathrm{A} h \Delta \mathrm{P}
$$

elle fournit :

$$
\frac{u_{2}-u_{1}}{u_{1}}=\left[\sqrt{1+2 \frac{\rho_{1}}{p_{p}} \frac{\Delta \mathrm{P}}{\rho_{1} u_{1}{ }^{2}}-1}\right]
$$

où $u_{1}$ et $u_{2}$ sont les vitesses de la particule à l'amont et à l'aval de la discontinuité ( $p_{1}, u_{1}$ densité et vitesse du fluide à l'amont). soit :

$$
\frac{\Delta u_{p}}{u_{p}} \simeq \frac{\rho_{1}}{\rho_{p}} \frac{\Delta \mathrm{P}}{\rho_{1} u_{1}^{2}}
$$

a) particule solide dans un $g a z \rho_{1} / \rho_{p} \sim 2.10^{-3}$ d'où avec :

$$
\begin{gathered}
\frac{\Delta \mathrm{P}}{p_{1} u_{1}^{2}} \sim 10 \\
\frac{\Delta u_{p}}{u_{p}} \sim 2.10^{-2}
\end{gathered}
$$

la particule solide ne subit pas d'accélération.

b) particule gazeuse dans un liquide

$$
\begin{gathered}
\rho_{1} / \rho_{p} \sim 0,8 \cdot 10^{3}: \\
\frac{\Delta u_{p}}{u_{p}} \sim 0,8.10^{3} \frac{\Delta \mathrm{P}}{\rho_{1} u_{1}{ }^{2}}
\end{gathered}
$$

alors que la variation de vitesse de fluide est $\Delta \mathrm{P} / p_{1} u_{1}{ }^{2}$. Les hypothèses simplificatrices du calcul ne sont pas vérifiées dans ce cas.

\subsection{Injection des particules dans le fluide [3].}

Ce calcul est classique et a été effectué par de nombreux auteurs, en particulier Lapple, Langmuir et Brun.

Lorsqu'une particule est injectée dans un fluide, elle n'est pas injectée en général suivant une ligne de courant; le phénomène est le même à l'aval d'une discontinuité de pression où les particules ont des vitesses et des directions différentes de celles de l'écoulement. En écoulement bidimensionnel, l'équation du mouvement de la particule est :

$$
\begin{aligned}
& \mathrm{M} \frac{d^{2} x}{d t^{2}}=-6 \pi a \mu \mathrm{D}_{0}\left[\frac{d x}{d t}-\mathrm{V}_{0}\right]+\mathrm{F}_{x} \\
& \mathrm{M} \frac{d^{2} y}{d t^{2}}=-6 \pi a \mu \mathrm{D}_{0} \frac{d y}{d t}+\mathrm{F}_{y}
\end{aligned}
$$

a l'instant zéro, les vitesses de la particule sont $(d x / d t)_{0},(d y / d t)_{0}$ avec une loi de résistance proportionnelle à la vitesse et des forces extérieures de composantes $\mathbf{F}_{i}, \mathbf{F}_{y}$ et les coordonnées sans dimension,

$$
\begin{aligned}
\xi & =x / \mathrm{V}_{0} \mathrm{~T} \\
\eta & =y / \mathrm{V}_{0} \mathrm{~T} \\
\tau & =t / \mathrm{T} \\
f_{x} & =\mathrm{F}_{x} \mathrm{~T} / \mathrm{MV}_{0} \\
f_{y} & =\mathrm{F}_{y} \mathrm{~T} / \mathrm{MV}_{0}
\end{aligned}
$$$$
\left(\mathrm{T}=\mathrm{M} / 6 \pi \alpha \mu \mathrm{D}_{0}\right)
$$

Les équations du mouvement sont :

$$
\begin{aligned}
& \frac{d^{2} \xi}{d \tau^{2}}+\frac{d \xi}{d \tau}-1-f_{x}=0 \\
& \frac{d^{2} \eta}{d \tau^{2}}+\frac{d \eta}{d \tau}-f_{y}=0
\end{aligned}
$$

si $\mathrm{D}_{0} \mathrm{~F}_{x} \mathrm{~F}_{y}$ sont constants on aura :

$$
\begin{aligned}
& \tilde{\xi}=\xi_{0}+\left(1+f_{0}\right) \tau+\left[\left(\frac{d \xi}{d \tau}\right)_{0}-1-f\right] \\
& \eta=\eta_{0}+f_{y} \tau+\left[\frac{d \eta}{d \tau_{0}}-f_{y}\right]\left[1-e^{-\tau}\right]
\end{aligned}
$$

ce qui permet de tracer des abaques en fonction de :

$$
\left(\frac{d \xi}{d \tau}\right)_{0} \cdot\left(\frac{d \eta}{d \tau}\right)_{0}, Y_{0} \ldots
$$

a) par exemple à l'aval d'un front de flamme :

$$
\begin{aligned}
\mathrm{V}_{0} & =10 \mathrm{~m} / \mathrm{s} \\
\mathrm{U}_{0} / \mathrm{V}_{0} & =0,2 \quad \mathrm{U}_{0}=2 \mathrm{~m} / \mathrm{s}
\end{aligned}
$$

angle d'entrée $15^{\circ}$ :

$$
\begin{aligned}
\rho_{p} & =2700 \mathrm{~kg} / \mathrm{m}^{3} \\
\frac{a}{h} \frac{\mathrm{S}}{\mathrm{D}_{0}} & =0,4 \quad \therefore \quad\left(\mu=6.10^{-4} \text { poises }\right)
\end{aligned}
$$


Pour $x=1 \mathrm{~cm}$ on obtient des écarts sur $y$ de :

-- $0,1 \mathrm{~cm}$ pour $a=10^{-3} \mathrm{~cm}$,

- 0,01 cm pour $a=2,5 \cdot 10^{-4} \mathrm{~cm}$;

ce qui conduit à ne prendre que des particules de diamètre inférieur à $2 \cdot 10^{-3} \mathrm{~cm}$.

b) exemple de bulles d'air dans l'eau :

$$
\begin{aligned}
\mathrm{V}_{0} & =10 \mathrm{~m} / \mathrm{s} & \\
\mathrm{U}_{0} / \mathrm{V}_{0} & =1 & \left(\mathrm{U}_{0}=\mathrm{V}_{0}\right)
\end{aligned}
$$

angle d'entrée $30^{\circ}$.

$$
\begin{aligned}
\rho_{p} & =1,3 \mathrm{~kg} / \mathrm{m}^{3}, \\
\rho_{f} & =1000 \mathrm{~kg} / \mathrm{m}^{3} \text { (particule sphérique), } \\
\mathrm{M} & =0,5 \mathrm{M}_{p}=0,5 \frac{4}{3} \pi \mathrm{a}^{3}, \\
\mu & =10^{-3} \mathrm{Pl}, \\
\mathrm{D}_{0} & \sim 1 .
\end{aligned}
$$

\begin{tabular}{|c|c|c|c|}
\hline$\xi$ & $x \quad$ In & $\eta$ & $m$ \\
\hline 0,5 & $0,55.10^{-2}$ & 0,11 & $0,12 \cdot 10^{-2}$ \\
\hline 1 & $1,1.10^{-2}$ & 0,17 & $0,19 \cdot 10-2$ \\
\hline 1,5 & $1,65.10^{-2}$ & 0,20 & $0,22.10-2$ \\
\hline 2 & $2,2.10-2$ & 0,22 & $0,24.10^{-2}$ \\
\hline
\end{tabular}

\begin{tabular}{|c|c|c|}
\hline$a$ & $10^{-4} \mathrm{~m}$ & $10^{-3} \mathrm{~m}$ \\
\hline $\mathrm{T}$ & $1,1.10^{-3}$ & $1,1.10^{-1}$ \\
\hline $\mathrm{V}_{0} \mathrm{~T}$ & $1,1.10^{-2}$ & 1,1 \\
\hline
\end{tabular}

Le calcul fournit les valeurs suivantes :

Au bout de $2,2 \mathrm{~cm}$, la particule s'est écartée de la ligne de courant initiale, dans la direction $\mathrm{O}_{y}$, de $2,5 \mathrm{~mm}$.

\subsection{Trajectoire des particules avec des lignes de courant courbes [3].}

Ces calculs sont les mêmes que ceux des problèmes de captation de particules ou de cyclones. Les équations du mouvement de la particule sont :

$$
\begin{gathered}
\mathrm{M} \frac{d^{2} r}{d t^{2}}-\mathrm{Mr}\left(\frac{d \theta}{d t}\right)^{2}=-6 \pi a \mu \mathrm{D}_{0}\left(\frac{d r}{d t}-\mathrm{V}_{r}\right) \\
-\frac{\mathrm{M}_{p} \rho}{r} \frac{\rho}{\rho_{p}} \mathrm{~V}_{\theta}^{2}+\mathrm{F}_{r} \\
\mathrm{Mr}^{2} \frac{d^{2} \theta}{d t^{2}}+2 \mathrm{Mr}\left(\frac{d r}{d t}\right) \frac{d \theta}{d t}=-6 \pi a \mu \mathrm{D}_{0} r \\
\left(r \frac{d \theta}{d t}-\mathrm{V}_{\theta}\right)+r \mathrm{~F}_{\theta}
\end{gathered}
$$

avec les coordonnées sans dimensions:

$$
\begin{aligned}
\tau & =t / \mathrm{T} \\
\lambda & =r / \mathrm{TV}_{0} \\
\omega & =\lambda^{2} \frac{d \theta}{d \tau}=\frac{r^{2}}{\mathrm{TV}_{0}^{2}} \frac{d \theta}{d t}
\end{aligned}
$$

et

$$
\begin{aligned}
\beta & =\frac{\mathrm{M}_{p}}{\mathrm{M}} \frac{\rho}{\rho_{p}} \\
v_{\theta} & =\mathrm{V}_{\theta} / \mathrm{V}_{0} \\
v_{r} & =\mathrm{V}_{r} / \mathrm{V}_{0}
\end{aligned}
$$

Les équations précédentes deviennent :

$$
\begin{aligned}
& \frac{d^{2} \lambda}{d \tau^{2}}+\frac{d \lambda}{d \tau}-\left(\frac{\omega^{2}}{\lambda^{3}}+\beta \frac{v_{\theta}^{2}}{\lambda}\right)-v_{r}-f_{r}=0 \\
& \frac{d \omega}{d \tau}+\omega-\lambda v_{\theta}-\lambda f_{\theta}=0
\end{aligned}
$$

qui peuvent se résoudre numériquement en fonction des données initiales et de $f_{r}$ et $f_{0}$.

En supposant que $D_{0}$ est constant et que les lignes de courant sont circulaires on écrit :

$$
\lambda=\lambda_{0}(1+\delta) \quad \delta \ll 1
$$

dans le cas où :

$$
\begin{gathered}
f_{\theta}=f_{r}=v_{r}=0 \quad \text { et } v_{\theta}=\left(\lambda_{0} / \lambda\right)^{n} \\
(n=1: \text { vortex })
\end{gathered}
$$

on obtient :

$$
\frac{d \omega}{d t}+\omega=\lambda_{0}(1+\delta)^{1-n}
$$

pour $n=1$ :

$$
\omega=\left(\omega_{0}-\lambda_{0}\right) e^{-\tau}+\lambda_{0}
$$

la solution de $\omega$ permet de trouver $\lambda$, qui peut s'écrire :

$$
\begin{aligned}
\delta= & \delta_{0}+\left(\frac{d \delta_{0}}{d \tau}\right)_{0} \tau+\left[k_{1}-k_{2} \delta_{0}-\left(\frac{d \delta_{0}}{d \tau}\right)_{0}\right] \\
& \frac{\tau^{2}}{r}+\ldots
\end{aligned}
$$

D'une façon plus simple, si on suppose que le terme d'accélération centrifuge est plus grand que le terme d'accélération radiale, les équations précédentes se réduisent à :

$$
\begin{gathered}
\frac{d \lambda}{d \tau}=\frac{\omega^{3}}{\lambda^{3}}+\frac{\beta v^{2} \theta}{\lambda} \\
\omega-\lambda v_{\theta}=0
\end{gathered}
$$


avec

$$
\begin{gathered}
v_{r}=0 \quad u_{\theta}=v_{\theta}, \quad \text { d'où } \\
u_{r}=\frac{d \lambda}{d \tau}=\left[1-\frac{1}{\mathrm{~S}} \frac{\rho}{\rho_{\nu}}\right] \frac{v_{\theta}^{2}}{\lambda} \\
v_{\theta}=\left(\lambda_{0} / \lambda_{\nu}\right)^{n}
\end{gathered}
$$

d'où

$$
\frac{\lambda}{\lambda_{0}}=\left[1+4\left(1-\frac{1}{S} \frac{\rho}{\rho_{D}}\right) \frac{\tau}{\lambda_{0}^{2}}\right]^{1 / 4}
$$

et

$$
\frac{r-r_{0}}{r_{0}} \sim\left[1-\frac{1}{\mathrm{~S}} \frac{\rho}{\rho_{p}}\right] \frac{\theta}{\lambda_{0}}
$$

soit :

$$
\frac{r-r_{0}}{r_{0}} \sim\left[1-\frac{1}{\mathrm{~S}} \frac{\rho}{\rho_{p}}\right] \frac{\theta \cdot \mathrm{V}_{0} \cdot \mathrm{M}_{p} \mathrm{~S}}{r_{0} 6 \pi a \mu \mathrm{D}_{0}}
$$

ou

$$
\frac{r-r_{0}}{r_{0}} \sim \frac{2}{9} \frac{V_{0}}{r_{0}}\left[1-\frac{1}{\mathrm{~S}} \frac{\rho}{\rho_{p}}\right] \frac{\mathrm{S} \cdot \rho_{p} \alpha^{2}}{\mu \mathrm{D}_{0}} \theta
$$

a) cas de l'air et d'une particule solide :

$$
\begin{aligned}
\mathrm{V}_{0} & =1 \mathrm{~m} / \mathrm{s}, \\
r_{0} & =0,01, \\
\mathrm{~s} & =1,002, \\
\rho & =1,3 \mathrm{~kg} / \mathrm{m}^{3}, \\
\mathrm{\rho}_{p} & =2500 \mathrm{~kg} / \mathrm{m}^{2}, \\
\mu & =1,510^{-5} \mathrm{Pl}, \\
a & =10^{-5} \mathrm{~m} .
\end{aligned}
$$

on obtient :

$$
\frac{\Delta r}{r} \sim 0,4 \theta, \text { soit, pour } \theta=\frac{\pi}{2} \quad \frac{\Delta r}{r}=0,6
$$

b) cas de l'eau et d'une particule solide :

$$
\begin{aligned}
& \mathrm{S}=1,002, \\
& \rho=1000 \mathrm{~kg} / \mathrm{m}^{3}, \\
& \mu=10^{-3} \mathrm{Pl} ;
\end{aligned}
$$

d'où :

$$
\begin{array}{ll}
\text { pour } \theta=\pi / 2 & \Delta r / r=0,5 \cdot 10^{8} a^{2}, \\
\text { pour } a=10^{-5} \mathrm{~m} & \Delta r / r=0,005, \\
\text { pour } a=10^{-4} \mathrm{~m} & \Delta r / r=0,5 .
\end{array}
$$

c) cas de l'eau et d'une bulle d'air :

$$
\begin{aligned}
& \mathrm{s}=0,5, \\
& \rho=1000 \mathrm{~kg} / \mathrm{m}^{3}, \\
& \rho_{p}=1,3 \mathrm{~kg} / \mathrm{m}^{2}, \\
& \mu=10^{-6} \mathrm{Pl}
\end{aligned}
$$

$$
\begin{aligned}
\text { d'où pour } \theta & =\frac{\pi}{2} & \frac{\Delta r}{r} & =-3,5 \cdot 10^{7} a^{2} \\
\text { pour. } a & =10^{-5} \mathrm{~m} & \Delta r / r & =-0,0035 \\
a & =10^{-4} \mathrm{~m} & \Delta r / r & =-0,35
\end{aligned}
$$

\subsection{Effet de la turbulence [4].}

On sait qu'on peut caractériser un écoulement turbulent par une longueur de corrélation $\lambda_{x}$ et, dans le cas d'un fluide où la vitesse moyenne est constante et parallèle à une direction fixe et où les fluctuations de vitesse sont faibles devant la vitesse moyenne, par une accélération instantanée de l'ordre de $\partial u u^{\prime} / \partial t$ dont la valeur quadratique moyenne est :

$$
\sqrt{\left(\overline{\left.\frac{\partial u^{\prime}}{\partial t}\right)^{2}}\right.}=\bar{u} \sqrt{\left(\overline{\left.\frac{\partial u^{\prime}}{\partial x}\right)^{2}}\right.}=\sqrt{2} \frac{\sqrt{\bar{u}^{2}} \cdot \bar{u}}{\lambda x}
$$

Lorsque $a \ll \lambda_{x}$, on peut considérer que la particule est à chaque instant dans un écoulement laminaire et, si $V_{p}$ est la vitesse de la particule et $V_{f}$ la vitesse du fluide, et soit $\gamma$ l'accélération du mouvement du fluide par rapport à des axes animés d'un mouvement de translation de vitesse égale à la vitesse moyenne du fluide, on a comme précédemment :

$$
\frac{4}{3} \pi \alpha^{3} \rho_{p} \frac{d \mathrm{~V}_{p}}{d t}=6 \pi \alpha \mu\left(\mathrm{V}_{f}-\mathrm{V}_{p}\right)
$$

d'où avec :

$$
\tau=\frac{2}{9} \quad \rho_{p} \frac{a^{2}}{\mu} \text { et } V_{p}=0
$$

pour $t=0$ :

$$
\mathrm{V}_{p}=\mathrm{V}_{f}+\gamma \tau\left[e^{-t / \tau}-1\right]
$$

l'écart entre la vitesse du fluide et la vitesse de la particule est au maximum égal à $\gamma \tau$ dont la moyenne quadratique est:

$$
\frac{2}{9} \frac{\rho_{p} a^{2}}{\mu} \sqrt{2} \frac{\sqrt{\overline{u^{\prime 2}} \cdot \bar{u}}}{\lambda_{x}}
$$

Le rapport des écarts quadratiques moyens est :

$$
\frac{2 \sqrt{2}}{9} \frac{p_{p} a^{2}}{\mu} \frac{\bar{u}}{\lambda_{\nu}}
$$

Les particules suivront donc le mouvement du fluide si :

$$
\begin{gathered}
\lambda_{x} \gg a \\
\frac{\rho_{n}}{\mu} a^{2} \frac{\bar{u}}{\lambda_{x}} \ll 1 \text { ou } \frac{a \bar{u}}{\nu} \frac{\rho_{\bar{\nu}}}{\rho_{f}} \frac{a}{\lambda_{x}} \ll 1
\end{gathered}
$$


Application. - On sait que dans la couche limite turbulente, si $u_{x}$ est la vitesse de frottement à la paroi, le rapport $\lambda_{x} u_{X} / \nu$ est fonction de $y u_{x} / \nu$; par suite : (1) s'écrit

$$
\frac{\lambda_{x} u_{\mathrm{X}}}{\nu} \gg \frac{a u_{\mathrm{X}}}{\nu}
$$

$\frac{\lambda_{x} u_{\mathrm{X}}}{\nu}$ à la paroi a un minimum de l'ordre de 5 , d'où $\frac{a u_{\mathrm{X}}}{\nu} \ll 1$

(2) s'écrit :

$$
\frac{\rho_{p}}{\rho_{f}} \cdot \frac{a^{2} u_{\mathrm{X}}^{2}}{v^{2}} \ll \frac{u_{\mathrm{X}}}{\bar{u}} \frac{\lambda_{, c} u_{\mathrm{X}}}{\nu}
$$

$\frac{u_{\mathrm{X}}}{\bar{u}} \frac{\lambda_{x} u_{\mathrm{X}}}{\nu}$ est fonction de $\frac{y u_{\mathrm{x}}}{\nu}$ et le minimum est de l'ordre de 1 , d'où la deuxième condition :

$$
\frac{a^{2} u_{x^{2}}}{\nu^{2}} \ll \frac{\rho_{f}}{\rho_{p}}
$$

Done deux conditions sont à remplir :

$$
\begin{aligned}
& \frac{a u_{X}}{\nu} \ll 5 \\
& \frac{a u_{X}}{\nu} \ll \sqrt{\frac{p_{f}}{p_{p}}}
\end{aligned}
$$

a) CAS DE L'AIR

$$
\begin{gathered}
\rho_{f} / \rho_{p} \ll 1 \sim 10^{-3}\left(\nu=1,5 \cdot 10^{-5} \mathrm{~m}^{2} / \mathrm{s}\right) \\
a u_{\mathrm{X}} \ll 5.10^{-7}
\end{gathered}
$$

$a_{X}$ est de l'ordre de $1 \mathrm{~m} / \mathrm{s}$ et par suite on doit avoir $a \ll 5.10^{-7} \mathrm{~m}$, soit $a \ll(1 / 2 \mu)$; à cette dimension, le mouvement brownien apparaît.

b) CAS D'UN LIQUIDE

$$
\left(\nu \sim 10-6 \mathrm{~m}^{2} / \mathrm{s}\right) \text { avec } \rho_{f} / \rho_{p} \sim 1
$$

il en résulte :

$$
a u_{\mathrm{X}} \ll 10^{-6}
$$

soit, avec $u_{\mathrm{X}}=0,1$ :

$$
a \ll 10^{-5} \mathrm{~m}=\frac{1}{100} \mathrm{~mm}
$$

c) CAS D'UN LIQUide AVEC $\rho_{f} / P_{p} \sim 1000$ :

$$
a u_{\mathrm{X}} \ll 10^{-6}, 30
$$

soit, avec $u_{\mathrm{X}}=0,1:$

$$
a \ll 3 \cdot 10^{-4}=\frac{3}{10} \mathrm{~mm}
$$

\section{3. - CONCLUSIONS}

Pour les écoulements dans les singularités ou les mouvements non permanents, les méthodes avec traceurs sont limitées par suite des écarts entre les trajectoires des particules et les éléments fluides; on a vu que:

- l'effet de masse ajoutée conduit à adopter des particules gazeuses, liquides ou solides dans les liquides; dans les gaz l'effet de masse ajouté est négligeable;

- dans une discontinuité de pression, les parti- cules solides ne sont pas déviées, contrairement aux particules fluides ou gazeuses;

- pour l'injection des particules traçantes, l'angle et la vitesse d'injection sont très importants;

-- sur des trajectoires courbes, les écarts $\Delta r / r$ sont proportionnels à la vitesse initiale et inversement proportionnels à la courbure;

- la visualisation des mouvements turbulents nécessite des particules de très petites dimensions $(1 / 100 \mathrm{~mm}$ à $0,5 / 1000 \mathrm{~mm})$.

\section{4. - REFERENCES BIBLIOGRAPHIQUES}

[1] J.M. Bourot. - Etude expérimentale des champs aérodynamiques. Actes dil Colloque International de Mécanique, Poitiers 1950 t. III, pp. 117 à 128 .

[2] H. Lamb. - Hydrodynamies. Cambridge University Press, $6^{\circ}$ édition 1959 , p. 123 .
[3] Battelle Memorial Institute. - Injection and combustion of liquid fuels. W.A.D.C. Technical Report 56-344, mars 1957.

[4] A. Fortier. - Mécanique des suspensions. Certificat d'Hydrodynamique Supérieure. Faculté des Sciences de l'Université de Paris, 1961-1962. 


\title{
ABSTRACT
}

\section{THE THEORETICAL LIMITATION OF VISUAL OBSERVATION METHODS}

\begin{abstract}
After reviewing the principal methods of visual observation used in fluid mechanics (i.e. optical methods, tracers, surface treatment) and their fields of application, the author establishes a few of the theoretical limitations of tracers on the basis of known research data on the mechanics of suspensions, especially in respect of centrifugal separation and dust-extraction problems.

In the examples considered, the added mass effect is allowed for and a certain number of simplifying assumptions are made to calculate the trajectories of the flow visualisation particles in pressure discontinuities, when injected into a fluid, in a curved-trajectory flow, and in a turbulent flow.

In a pressure discontinuity, the fluid flow deviates, but the solid particles undergo practically no deflection. It is also seen that the proposed theoretical pattern does not hold good in the case of gas particles.

Solid particles injected from an oblique angle into a flow will, if smaller than $0.02 \mathrm{~mm}$ in size, quickly take up the same direction as the fluid trajectories.

Numerous theoretical solutions have already been put forward for the curved-trajectory flow case; they show that the error on the particle trajectory radius vectors is directly proportional to the initital velocity and inversely proportional to the curvature.

In order to allow the turbulence in the turbulent flow case to be schematized, particles sizes should not exceed one micron for a gas and $0.01 \mathrm{~mm}$ for a liquid, if the particles are to follow the turbulent fluctuations of the fluid.
\end{abstract}

\section{ISCUSSION}

Président : M. Chapoutriek

M. le Président remercie M. Faune de sa communication, qu'il a trouvée un peu pessimiste parce qu'elle aurait pour résultat, dans une certaine mesure, d'infirmer ce qui a été présenté avant. Se tournant vers nos amis qui ont animé cette réunion, il leur demande si l'on s'est préoccupé des limites de validité, soit pour l'ébullition, soit pour des phénomènes de visualisation aérodynamique.

M. le Président rappelle que les conférenciers précé dents ont dit qu'ils ne s'étaient pas bornés à des observations qualitatives et qu'ils avaient effectivement fait des mesures, donné des tracés, etc. On observe sur certaines figures projetées que les bulles ont un petit segment : on doit donc pouvoir mesurer le champ de vitesse. Les limites indiquées par M. FAUre sont-elles respectées? Ce champ de vitesses tracé par les éléments traçants correspond-il à celui de la réalité?

M. Wentó indique qu'il a un peu escamoté la difficulté, grâce à la disposition verticale du tunnel O.N.E.R.A. et à la production des bulles d'air au moment du remplissage du réservoir après introduction dans l'eau d'un agent émulsionnant. En effet, les bulles d'air obtenues dans ces conditions remontent dans le réservoir plus ou moins vite suivant leur diamètre. A 1 ia mise en vitesse de l'écoulement, seules les bulles les plus fines sont rattrapées et entrent dans le champ de visualité. Le diamètre de ces bulles est de l'ordre du $1 / 20^{\circ}$ de $\mathrm{mm}$ et leur vitesse de remontée de l'ordre $\mathrm{du} \mathrm{mm} / \mathrm{s}$, ce qui est négligeable devant la vitesse de $10 \mathrm{~cm} / \mathrm{s}$ de l'éconlement extérieur, e'est-à-dire en dehors du voisinage immédiat des maquettes.
Cependant, même dans cette partie de l'écoulement, les bulles d'air foumissent des renseignements, et indiquent notamment le caractère de la couche limite :

- absence ou réfraction des bulles en cas de laminarité;

- trajectoires désordonnées des bulles en cas de turbulence, et de là, localisation de la transition et allure des lignes de courant moyennes dans la région turbulente.

M. le Président demande, étant donné le domaine où l'on se place dans ce cas, si la critique de M. FaUne est à peu près satisfaite.

M. LEgENDRE estime que les bulles d'air utilisées a l'O.N.E.R.A. pour rendre visible l'écoulement dans l'eau n'altèrent pas dangereusement cet écoulement. Avant le début d'une expérience, il est loisible de vérifer que les très petites bulles d'air sont pratiquement immobiles. L'observation en cours d'expérience ne révèle pas de coalescence importante. Enfin, les trajectoires des bulles sont pratiquement indépendantes de leur diamètre, même dans le voisinage des points de décollement, où les vitesses sont faibles.

M. Faune précise que e'est d'ailleurs dans le cas des bulles d'air dans l'eau que les écarts sont les plus faibles. Ils seraient plus importants dans le cits des particules solides dans l'air ou rans l'eau.

M. le Président est rassuré de voir que ce que M. Faure a dit n'annule pas ce que l'on a vu.

Sur une demande de M. Legendre, M. Favre indique que le calcul qu'il a exposé est un calcul original de I. le Professeur Fontien et que si l'on n'a pas vu intervenir les fréquences, e'est que l'on a donné un schéma 
très simplifié pour montrer qu'il serait pratiquement impossible de visualiser.

M. SÉMÉnia voudrait ajouter quelques mots sur les problèmes de masses entraînées.

La masse entrainée d'une sphère est à peu près de 0,5 fois le volume du produit déplacé. Pour les petites sphères, it n’y a qu'une étude d'un Japonais $\left(^{\star}\right)$ faite en 1925; celui-ci a trouvé des masses ajoulées beaucoup plus faibles lorsque le diamètre diminue. Malheureusement, il n'est pas descendu très loin ( $1 \mathrm{~mm}$ environ) et son travail a été controversé.

Pour des paillettes d'aluminium il faut considérer, non plus la masse, mais la surface ou un produit de la hauteur par le diamètre de la paillette.

Suivant une remarque de M. SÉméRIA, M. Faure indique que dans le cas d'une variation brutale de pression pour laquelle la variation de vitesse est plusienrs centaines de fois celle du fluide, les approximations précédentes ne sont plus bonnes.

M. SÉméria a remarqué que dans le livre de Prandtl: «Introduction à la mécanique des fluides », on trouve seulement le calcul pour les accélérations : si on prend une bulle d'air de masse négligeable, l'accélération de la bulle est trois fois au maximum celle du liquide.

Certaines personnes ont pensé à un effet Magnus sur les bulles qui sont dans un gradient de vitesse et qui se mettent à tourner en s'éloignant des parois. Ccci peut être aussi une cause de défaut de visualisation.

M. Faure mentionne que les autres grandeurs varient entre 0,45 et 0,55 , ce quj ne change pas les ordres de grandeur.

M. SCḾrRia objecte, tontefois, que, dans l'expérience japonaise, on descend jusqu'à 0,1 . Mais les résultats ne sont pas très précis et M. Striéris n'ose pas les défendle.e.

M. Willm demande des précisions sur ce qui se passe lorsque la particule injectée a exactement la densité du liquide dans lequel on l'injecte. Ce cas doit présenter un grand intérêt, et dans ces conditions, il semble que la représentation de l'écoulement ne puisse être perturbée par des phénomènes d'inertie, et reste correcte même en présence de fortes courbures ou accélérations. En particulier, la notion de masse entraînée ne doit

(*) O. Mryagy. - Phil. Mag., S. 6, vol. 50, n॰ 295, pp. 112-140, $\left({ }^{*}\right)$ plus s'appliquer, tout ceci dans la mesure, tout au moins, où les dimensions de la particule sont petites vis-à-vis des gradients de vitesse.

M. Faure précise que, dans ce cas, toutes les écuations s'écrivent, mais que le terme M qui intervient dans le coefficient d'inertie est égal à la somme de la masse de la particule et de la masse ajoutée. Ce résultat a été établi, en 1840 ou 1850 , par DU BUAT et Lamis. Les questions de masses ajoutées ne sont valables que dans un très petit nombre de cas.

M. Mexer pense que les équations de M. Faure s'appliquent quand les densités sont suffisamment différentes. Le cas que vient d'évoquer M. WILIM nécessiterait une autre étude, et les choses se présenteraient comme suit :

- Si on remplace une goutte d'un fluide 1 par une goutte d'un fluide 2 ayant rigoureusement les mêmes caractéristiques, la goutte suit évidemment l'écoulement, contrairement à ce qu'indiquent les équations de M. FaUre, dans lesquelles il manque un terme qui n'est plus négligeable dans ce cas.

- Si, seules les densités sont identiques, trois phénomènes supplémentaires, non compris dans l'étude de M. FAURE, apparaissent :

10 Dans tous les écoulements :

- la tension superficielle fournit un travail quand la bulle se déforme sous l'effet de l'écoulement;

- la différence de viscosité intervient, et si la bulle est très visqueuse, on obtient le phénomène décrit dans la thèse du grand Einstein.

$2^{\circ}$ En écoulement turbulent, la tension superficielle empêche la bulle d'être disloquée et change complètement l'écoulement à l'échelle de la bulle, done aussi l'écoulement moyen.

Dans tous les cas, l'étude de M. Faure pourrait, en principe, être complétée, mais ne pourrait alors plus énoncer des résultats généraux simples.

M. Faure signale que les remarques de M. Meyer s'appliquent au cas de particules fluides dans un fluide, et non au cas de particules solides dans un fluide.

M. le Président est maintenant obligé de lever la séance et remercie encore tous les participants et particulièrement nos amis de l'O.N.E.R.A., de l'Institut Francais du Pétrole et du Centre Nucléaire de Grenoble, qui ont beaucoup animé la séance et ont introduit les auditeurs dans un univers qui les a passionnés tous.

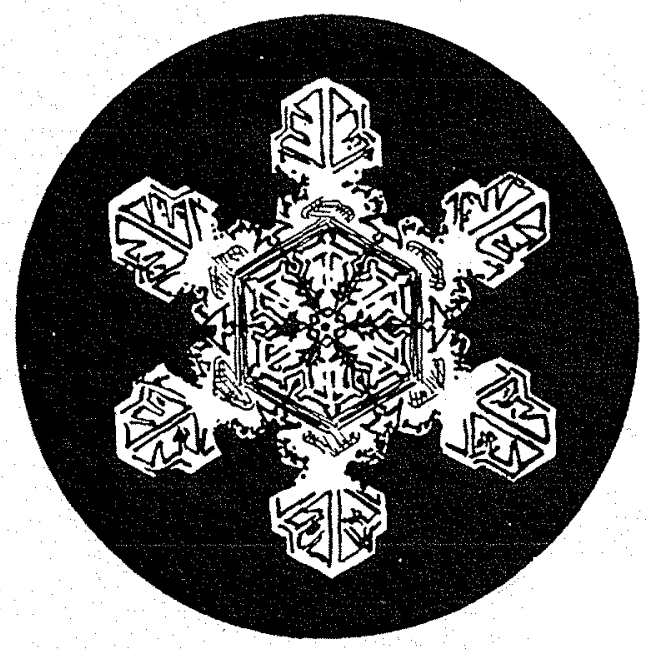

